\title{
Rehabilitation therapy in patients with psoriatic arthritis after SARS-CoV-2 infection
}

\author{
Diana Maghiar', Nicoleta Anamaria Pascalau ${ }^{2,4}$, Liviu Lazar ${ }^{1,2,3}$ \\ ${ }^{1}$ Doctoral Studies Department, Biomedical Science, Oradea, Romania \\ ${ }^{2}$ Department of Medicine-Psycho-Neuroscience and Recovery, Faculty of Medicine and Pharmacy, Oradea, Romania \\ ${ }^{3}$ Baile Felix Medical Rehabilitation Hospital, Baile Felix Resort, Bihor County, Romania \\ ${ }^{4}$ Pelican Hospital, Oradea, Romania
}

\begin{abstract}
Objectives. We want to present the evolution of a lot of patients, previously diagnosed with psoriatic arthritis, who last spring went through a difficult period due to infection with the new coronavirus. After healing from COVID-19, the patients had returned to the hospital after a period of 4-6 months, to follow a rehabilitation treatment, the majority of the accusations being those related to psoriatic arthritis, with close follow-up of these patients in connection with the treatment applied.
\end{abstract}

Material and methods. The patients were evaluated at hospitalization (biological inflammatory markers like CRP and ESR), pain scale, DAPSA score, PASI and the quality of life score (DLQI and QOL scale), after which they followed different rehabilitation treatments for a period of 21 days. After 3 months of completing this treatment they were re-evaluated. Patients diagnosed with psoriatic arthritis who do not have documentation to suggest SARS-CoV- 2 infection (antibodies/ previous RT-PCR positive tests) were not included in the study.

Outcomes. There were some significant differences in terms of the initial score at hospitalization and that performed after rehabilitation treatment. Most of the indices performed had lower values at reassessment (pain scale score, DAPSA, PASI, DLQI and even lower values of CRP and ESR), thus resulting in an important step in terms of the beneficial effects of rehabilitation therapy, both for patients with psoriatic arthritis and for post-COVID-19 recovery. The most important change was observed in the score for quality of life.

Conclusions. The inclusion of rehabilitation therapy in patients with psoriatic arthritis should be a step that each patient should take. Its effects are long-term, with periods of pain decreasing in frequency and intensity, thus changing the quality of life of these patients. The mental, social and emotional impact of COVID-19 on people who have gone through the disease can be changed in a good way, also following a rehabilitation therapy.

Keywords: COVID-19, rehabilitation therapy, psoriatic arthritis, psoriasis

\section{Abbreviations}

SARS-CoV-2 - severe acute respiratory syndrome coronavirus 2

COVID-19 - coronavirus disease 2019

PsA - psoriatic arthritis

DAPSA - disease activity in psoriatic arthritis

PASI -psoriasis area and severity index
DLQI - dermatology life quality index

QOL scale - quality of life scale

CRP - $C$ reactive protein

ESR - erythrocyte sedimentation rate

\section{INTRODUCTION}

For a year and a half, life around the world has been on the break due to the appearance of the new coronavirus. Following this pandemic, many of the

chronic patients suffer more due to the blockage of the medical system in the mission to stop SARSCoV-2 infections and to treat infected patients. Those who suffered the most were obviously those 
suffering from chronic diseases, for which the controls were reduced, and the fear of calling doctors increased side by side with the number of new infections.

Psoriatic arthritis is chronic inflammatory arthritis associated with psoriasis and found in about 20 to $30 \%$ of such patients. It shares many clinical features with other spondylo-arthropathies and also rheumatoid arthritis (1). PsA patients have heterogeneous clinical presentations, with diverse articular and dermatological features and varied disease courses and outcomes. PsA was initially considered to be a mild disease, but in the past decade, $40 \%-60 \%$ of patients have developed erosive and deforming joint complications. PsA-induced joint damaging complications not only led to lower articular function and higher mortality but also affect patients' ability to work and affect their social relationships (2-4).

As of the autumn of 2020, the coronavirus disease-2019 (COVID-19) pandemic continues to persist with over 44 million confirmed cases globally. An early recognized vulnerable group were patients with autoimmune diseases such as rheumatoid arthritis, and sero-negative spondyloarthropathies, as they require immune modulation therapies to control their disease severity (5).

Patients with rheumatic disease are at increased risk of infection. This is due to a combination of underlying disease and treatment-related factors. It was within this context that, at the beginning of the COVID-19 pandemic, concerns arose regarding the potential consequences of SARS-CoV-2 infection in patients with rheumatic and autoimmune diseases. Given the potential negative effects of immunosuppressive drugs on viral clearance, there was concern that infection would be associated with increased COVID-19 severity and excess mortality in patients with rheumatic disease $(6,7)$.

The main object of this study was to observe the differences between the evolution of patients before and after rehabilitation treatment post-COVID-19, taking into account of course the underlying disease, in this case being psoriatic arthritis. Thus, in this study we took into account scores such as DAPSA, PASI for evaluating the evolution of arthropathic disease, the quality of life score for both PsA (DLQI) and post-SARS-CoV-2 infection (QOLs). As important biological markers in assessing inflammatory status, we studied the evolution of CRP and ESR. Because the most important complaint of the patients was the joint pain, the pain scale was taken into account both at hospitalization and at re-evaluation after rehabilitation treatment.

\section{MATERIALS AND METHODS}

We managed to put together a lot of 48 patients with psoriatic arthritis who were hospitalized 1 year ago due to SARS-CoV-2 infection. COVID-19 manifestations ranged from moderate to severe in some cases. After discharge, these patients presented themselves at the Clinical Hospital of Rehabilitation to undergo rehabilitation treatment, with the reported disease manifestations being mostly articular (joint pain with different degrees of intensity, loss of joint function that had major impact on daily activities and even sleep disorders, in some cases depression).

Standard assessments for patients consisted of general data such as date of birth, sex, place of origin, school and socio-economic status, weight, height. Other important data used were the history of the disease, comorbidities and the medication used. Patients diagnosed with psoriatic arthritis who do not have documentation to suggest SARSCoV-2 infection (antibodies/previous RT-PCR positive tests) were not included in the study.

From the point of view of the disease that interests us as we used the PASI score - psoriasis area and severity index; DAPSA score - disease activity in psoriatic arthritis; DLQI - dermatology life quality index and even QOLS - quality of life scale. As biological markers, which are the most useful in our case, we used the inflammatory ones: C-reactive protein and erythrocyte sedimentation rate. Also we included the comparison data between the average value on pain scale at admission and again at the re-evaluation.

The patients underwent thermal treatment (hydrotherapy), therapeutic massage and kinetotherapy for 21 days at the Clinical Hospital of Rehabilitation Băile Felix. According to doctors, thermal water from Băile Felix can be used both in the internal cure (crenotherapy) for certain diseases of the digestive tract, stomach, small and large intestines, gallbladder, metabolic and endocrine disorders and in the external cure (bathing). All patients were evaluated initially, when they were admitted to hospital and after 3 months. In addition, we had access to the data provided after their hospitalization during SARS-CoV-2 infection.

Patients benefited from 3 sessions of different recovery therapies. Thermal water treatment (thermal springs in Băile Felix, with a natural thermality of 3738 degrees Celsius, the chemical composition defines it as a mesothermal oligomineral water. Among the anions in the order of the values found the most important are: bicarbonate (HCO3-) - $439 \mathrm{mg} / \%$, sulfate (SO4 2-) - $208 \mathrm{mg} \%$, and of the most important cations are: calcium - $139 \mathrm{mg} / \%$ sodium $-71 \mathrm{mg} / \%$, magnesium - $24 \mathrm{mg} / \%$ ). Hydrotherapy included sessions like shot steam baths, underwater showers, individu- 
al and group sessions of physical therapy in water, mobilisations in the pool, warm baths, foot and hand showers. Massage may be beneficial in psoriatic arthritis because it can help decrease pain and promote healthy circulation in the body. Several types of massage techniques were used (from Swedish massage to deep tissue massage) and they were selected according to the each patient. In terms of kinetotherapy, depending of the joint that is affected, various types of exercises were performed, so as not to negatively influence mobility and pain (therapeutic exercise, recreational exercise).

The data used in this study was approved by the hospital's ethics committee and it meets all the requirements of the National Statement on Ethical Conduct in Human Research (Helsinki Declaration, revised 2013).

\section{OUTCOMES}

In relation to the sex of the patients, most of them were female $(>50 \%)$, thus confirming the data from the specialty literature according to which women are more affected by psoriatic arthritis than men. The age of the patients in the study group varies between 43 and 72 years, with an average age of 62.17. Most frequently affected was the category aged between 55-65 years (42.7\%). Depending on the treatment of psoriatic arthritis, $39.58 \%$ of patients used biologically targeted therapies, the rest being on the classic treatment with immunosuppressants (in some cases the treatment was temporarily stopped during SARS-CoV-2 infection).

Depending on the joint affection, it will be noticed that the most commonly affected joint is the distal inter phalangeal joint of the hands (75\%), followed by the sacroiliac joint $(25 \%)$. The onset of joint symptoms was 3-4 years after the diagnosis of psoriasis, in some cases fewer than 3 years. Patients over the age of 60 years were of course more affected. The influence of SARS-CoV-2 virus infection and damage to the immune system has led to these patients experiencing much more frequent exacerbations than before infection. The patients included in the study group suffered moderate and even severe forms of COVID-19. During treatment for SARS$\mathrm{CoV}-2$, for some patients it was decided to temporarily stop basic treatment for psoriatic arthritis, due to their immunosuppressive effect, which was resumed after resolution of the infection, if the patient's condition allowed it.

Over $50 \%$ of patients complained of severe joint pain, on a scale of 1-10 with an average of 7.2, which no longer yields to usual painkillers, some of them even receiving those based on opioids. Before starting the rehabilitation treatment, we performed the PASI, DAPSA and the quality of life score. In addition to these data, we also considered CRP and ESR values. Evaluation of inflammatory syndrome based on CRP, reveals elevated values before the start of rehabilitation treatment, with an average value of 37.1 $\mathrm{mg} / \mathrm{dl}$ (laboratory reference value was $<0.5 \mathrm{mg} / \mathrm{dl}$ ). ESR value at hospitalization was increased, in both sexes, with an average value of $42.3 \mathrm{~mm} / \mathrm{h}$.

The next evaluation was performed 3 months after the hospitalization for the rehabilitation treatment. The first major change observed was in relation to the pain scale. If at admission we had an average value of 7.2 (on a scale of 1-10), 3 months after the end of recovery therapy, we end up with an average value of 4.3. $(E S=0.89)$.

The data provided was used in the sense of decreasing the CRP values as well as the ESR values, with an average value of $7.8 \mathrm{mg} / \mathrm{dl}$ for CRP and an average value of $8.2 \mathrm{~mm} / \mathrm{h}$ of ESR. $(\mathrm{p}<0.05)$

The initial PASI score was graded at a maximum of 18 with an average value of 12.87, reflecting that most patients have moderate to severe disease activity. At the subsequent evaluation, the maximum PASI score reaches a score of 14 points, thus no longer having a category of patients with severe disease activity. $(p<0.05$, ES = 0.78).

The baseline value of the DAPSA score revealed $62.5 \%$ of patients with moderate impairment, however, there are cases with a maximum score of over 28 (maximum initial value 31 ) denoting the severity of the disease. However, after 3 months, a decrease in the DAPSA score was observed in more than half (75\%) of cases, while for the remaining $25 \%$ the score remained unchanged. The new maximum score after rehabilitation therapy reached 26 , so most patients fall into the category of moderately affected $(\mathrm{p}<0.05, \mathrm{ES}=0.81)$.

Probably the biggest differences between the initial values and those performed later, were in the score for quality of life. The situation of the pandemic plus fact that these patients went through a new disease, with rather severe damage to their health, implicitly affected the quality of life of patients, without taking into account the new exacerbations due to a previous disease. Dermatology life quality index (DLQI) is the most commonly applied measure of health-related quality of life (HRQoL) in psoriasis patients. It is among the defining criteria of moderate-to-severe psoriasis and present in treatment guidelines. At admission, the maximum DLQI score accumulated a score of 22 (extremely high impact on quality of life), observed in a patient with a DAPSA score of 31, who complained of joint pain at least 3 weeks before. Some of the patients also benefited from psychological therapy, given the increased emotional and mental impact they were subjected to. However, the good news comes at the next evaluation, after they finished the rehabilitation therapy, when from the maximum score of 22 DLQI, 
a new value is reached, a lower one, with a maximum of 17 which, while it has an improved effect on the patient's life, still represents an important step, by confirming the fact that rehabilitation therapy has an effect on the quality of life, in some cases even significantly $(p<0.001$, ES-1.32) (table 1).

From the point of view of QOL scale (16 items, minimum-16, maximum-112), at hospitalization, there were cases with minimum values of up to 34 , with an average value of 58.25. After rehabilitation therapy, the new minim value reached 62 , with an average of 73.7 (table 2).

TABLE 1. Evolution of DLQI score

\begin{tabular}{|l|c|}
\hline \multicolumn{1}{|c|}{ DLQI } & \multicolumn{1}{c|}{ Study group } \\
\hline Min-Max & $10-22$ \\
\hline M $\pm S D$ & $12.04 \pm 1.48$ \\
\hline \multicolumn{2}{|c|}{ At 3 months } \\
\hline Min-Max & $6-17$ \\
\hline M $\pm S D$ & $8.59 \pm 2.42$ \\
\hline p & $<0,001$ \\
\hline ES & $\mathbf{1 . 3 2}$ \\
\hline
\end{tabular}

TABLE 2. Evolution of QOL score

\begin{tabular}{|l|c|}
\hline \multicolumn{1}{|c|}{ QOL } & \multicolumn{2}{c|}{ Study group } \\
\hline Min-Max & $34-75$ \\
\hline M \pm SD & $58.25 \pm 1.8$ \\
\hline & At 3 months \\
\hline Min-Max & $62-98$ \\
\hline M \pm SD & $73.7 \pm 2.41$ \\
\hline $\mathbf{p}$ & $<0,001$ \\
\hline ES & $\mathbf{1 . 2 7}$ \\
\hline
\end{tabular}

\section{DISCUSSION}

Although this study was performed on a small group of patients, the data obtained from it may be useful, with recovery therapy having a huge advantage, both in terms of disease progression as well as mentally and socially for the patient, so that in the future studies on recovery therapy can be performed on a larger group of patients. The improvements observed in the different disease assessment scores, as well as the decrease of the values of the inflammatory markers represent an important step for the future in the use of the rehabilitation therapy.

Just like our study, the study performed by Peter et al. (8) showed that patients with psoriasis and PsA are showing improvements in the CRP levels and PASI score after 3-week-long rehabilitation. Zhu et al. (9) concluded post-COVID rehabilitation therapy, regardless of patients' associated diseases not only reduces mortality rate, hospital admission but also saves medical resource, therefore rehabilitation therapy should be introduced into the mainstream treatment of COVID-19.

\section{CONCLUSIONS}

The last year has affected the world's population, from small to large, with the development of this pandemic caused by the SARS-CoV-2 virus. The sudden change in lifestyle affected especially the elderly and the chronically ill, the health system being blocked by people with COVID-19. Post-infection consequences with SARS-CoV-2 will begin to be present in the near future, in some cases already being observed.

Patients belonging to this study passed through this terrible COVID-19 pandemic period and developed frequent exacerbations of psoriatic arthritis within 4-6 months after healing. The most common complaints were of course the pain located in different joints that does not respond to common painkillers, as well as the loss of joint function. This has a major impact on the patient's quality of life, on daily life activities, on social life and workplace. They underwent rehabilitation treatment, consisting of several procedures designed to relieve their pain and of course joint recovery, like thermal bath therapy, kinetotherapy and therapeutic massage, all established individually for each patient by the specialist, depending also on their comorbidities.

Rehabilitation therapy had a major impact especially on the quality of life of these patients, with decreases in quality of life score values compared to the initial values. The same can be said about the PASI and DAPSA score, when re-evaluated, a decreased score was observed. As for the inflammatory markers, evaluated by CRP and ESR testing, we can say that rehabilitation therapy has its effect, of course correlated with the basic drug treatment of the disease, because lower values are observed in evaluations performed after rehabilitation therapy. Last but not least, the main complaint of the patients, namely the intense joint pain, decreases in intensity at the end of the recovery treatment, as well as at the subsequent evaluation.

Of course, further studies with a larger group of patients would continue to be helpful in further highlighting the benefits of rehabilitation therapy. It seems that the rehabilitation therapy can positively influence the life of patients not only with psoriatic arthritis, but also after COVID-19.

\section{Note}

All authors contributed equally to the manuscript and read and approved the final version of the manuscript. 


\section{REFERENCES}

1. Tiwari V, Brent LH. Psoriatic Arthritis. 2021 Aug 11. In: StatPearls [Internet]. Treasure Island (FL): StatPearls Publishing; 2021 Jan.

2. Slobodin G, Rosner I, Rozenbaum M, Boulman N, Kessel A, Toubi E. Psoriatic arthropathy: where now? Isr Med Assoc J. 2009 Jul;11(7):430-4.

3. Palmer D, El Miedany Y. Early psoriatic arthritis: facing the challenge. Br J Nurs. 2013 Sep 12-25;22(17):1014-20.

4. Helliwell PS, Mease PJ, FitzGerald O, Taylor WJ, van der Heijde D. Peripheral spondyloarthritis and psoriatic arthritis; overlaps and distinctions: a report from the GRAPPA 2012 annual meeting. J Rheumatol. 2013 Aug;40(8):1446-9.

5. Conway R, Konig MF, Graef ER, Webb K, Yazdany J, Kim AHJ. Inflammatory arthritis in patients with COVID-19. Trans/ Res. 2021 Jun;232:49-59.

\section{Conflict of interest: none declared} Financial support: none declared
6. Au K, Reed G, Curtis JR. High disease activity is associated with an increased risk of infection in patients with rheumatoid arthritis. Ann Rheum Dis. 2011;70:785-791.

7. Price E, MacPhie E, Kay L, Lanyon P, Griffiths B, et al. Identifying rheumatic disease patients at high risk and requiring shielding during the COVID-19 pandemic. Clin Med (Lond). 2020 May 5;20(3):256-61.

8. Péter I, Jagicza A, Ajtay Z, Boncz I, Kiss I, Szendi K, Kustán P, Németh B. Balneotherapy in Psoriasis Rehabilitation. In Vivo. 2017 Nov-Dec;31(6):1163-1168.

9. ZhuY, Wang Z, Zhou Y, Onoda K, Maruyama H, Hu C, LiuZ. Summary of respiratory rehabilitation and physical therapy guidelines for patients with COVID-19 based on recommendations of World Confederation for Physical Therapy and National Association of Physical Therapy. J Phys Ther Sci. 2020;32(8):545-549. 